\title{
A NOTE ON UNARY ALGEBRAS
}

JOHN MARICA

In [1] the possibility of proving unique factorization theorems for some classes of unary algebras was not discussed. We offer an example to show that even in a very restricted class of algebras, an algebra may have two distinct "prime" factorizations.

A unary algebra (i.e., a set $X$ and a function ', on $X$ into $X$ ) is called basic if it is connected and has a one element subalgebra. A constant function corresponds to a basic algebra of degree one. It is easily seen that basic algebras of degree one behave under Cartesian product in the same way that positive integers do under the usual product, and that consequently one has unique factorization for this class of algebras.

The following four basic algebras are of degree two, are indecomposable as Cartesian products, and no two are isomorphic. A direct calculation shows that $A \times B \cong C \times D$.

$$
\begin{aligned}
& A=\left(A,,^{\prime}\right) ; A=\{a, b, c, d\},,^{\prime}=\{(a, c),(b, c),(c, d),(d, d)\} \\
& B=\left(B,,^{\prime}\right) ; B=\{a, b, c\},{ }^{\prime}=\{(a, c),(b, c),(c, c)\} \\
& C=\left(C,{ }^{\prime}\right) ; C=\{a, b, c, d, e, f\}, \\
& \qquad \quad=\{(a, d),(b, d),(c, d),(d, f),(e, f),(f, f)\} \\
& D=\left(D,,^{\prime}\right) ; D=\{a, b\},,^{\prime}=\{(a, b),(b, b)\} .
\end{aligned}
$$

\section{REFERENCE}

1. John G. Marica and Steven J. Bryant, Unary algebras, Pacific J. Math. 10 (1960), 1347-1359.

\section{Sacramento State College}

Received by the editors June 14, 1962. 